Research Article

\title{
Online Static Voltage Stability Monitoring for Power Systems Using PMU Data
}

\author{
Jianhong Pan, Aidi Dong, Jiashu Fan, and Yang Li iD \\ State Grid Jilin Electric Power Company Limited, Changchun 130000, China \\ Correspondence should be addressed to Yang Li; liyang@neepu.edu.cn
}

Received 28 November 2020; Revised 11 December 2020; Accepted 16 December 2020; Published 29 December 2020

Academic Editor: Bo Yang

Copyright (C) 2020 Jianhong Pan et al. This is an open access article distributed under the Creative Commons Attribution License, which permits unrestricted use, distribution, and reproduction in any medium, provided the original work is properly cited.

\begin{abstract}
A new online static voltage stability monitoring method for power systems is proposed by using phasor measurement unit (PMU) data in this paper. This approach uses the real-time power, voltage, and phase angle data collected by the PMU to estimate the power flow Jacobian matrix of the system, and then the static voltage stability is monitored via the minimum singular values (MSVs) of the power flow Jacobian matrix. The novelty of the approach lies in the fact that it only utilizes PMU data for implementing online monitoring of the power system static voltage stability, independent of the physical model and its parameters. The application results on the IEEE 57-bus test system verify the effectiveness of the proposed approach.
\end{abstract}

\section{Introduction}

Voltage stability assessment (VSA) has been recognized as an important task to ensure the secure and economical operation of power systems [1,2]. Problems arising from the growing integration of intermittent renewable power generation in a variety of forms such as active distribution networks [3] and microgrids [4,5] and integrated energy systems [6] are nudging power systems toward potential dynamic instability scenarios due to the inherent uncertainties of renewable generation [7]. As a new generation of DC transmission technique, voltage source converter-based high voltage direct current (VSC-HVDC) has become a popular opinion of power transmission due to its significant advantages such as independent adjustments of active and reactive powers [8], asynchronous interconnection between islands [9], and black-start capability [10]. In addition, the growing integration of energy storage [11], electric vehicles [12], cyber attacks [13-15], and increasingly diversified demands $[16,17]$ affect the stable operation of the system to a certain extent. All these shifts pose new challenges in maintaining the system working reliably and securely. In recent years, the wide-area measurement system (WAMS) using time-stamped phasor measurement units (PMUs) has been receiving ever increasing attention from both academia and industry, which makes it possible to explore wide-area protection and control (WAPaC) schemes to avoid the system collapse [18-21]. Therefore, the voltage stability monitoring and assessment of power systems based on PMU data are of great significance in the new context [22-25].

Since the first $\mathrm{dQ} / \mathrm{dU}$ criterion proposed by the Soviet scholar N.M in the 1940s, a large number of voltage stability analysis approaches of power systems have been developed, such as the sensitivity method [26], continuous power flow method [27], the singular value decomposition method [28], and so on. The sensitivity method is only suitable for simple power systems, and sometimes there are discriminant errors when it is used in multimachine systems. In theory, the feasible solution domain method can calculate the voltage stability margin of a given operation mode, but the calculation of critical injection vectors involves complex nonlinear problems, and the computation is heavy. The multivalue method of power flow needs to track and calculate the multisolution value of the injection quantity repeatedly, and the static stability margin of the system voltage is approximate. References [29-31] illustrate the feasibility of the singular value decomposition theory of the trend Jacobian matrix, but the above methods need to know the 
topology and parameters of the system and cannot monitor the static voltage stability of the grid online. The widespread deployment and successful application of PMUs break through this bottleneck and shed new light on online monitoring of the static voltage stability of power systems [32-34].

In recent years, PMU data have been successfully used for real-time power system stability assessment due to its powerful data acquisition capabilities. In [35], an extreme learning machine- (ELM-) based transient stability assessment (TSA) model optimized by improved particle swarm optimization is proposed by using PMU data; to address the issue of lacking online learning ability in current methods, an online sequential ELM- (OS-ELM-) based TSA method is presented for power systems in [36]; a feature selection approach is presented for TSA based on improved maximal relevance and minimal redundancy criterion and PMU measurements in [37]; a new online learning mechanism based on an ensemble of OS-ELM (EOS-ELM) is developed for TSA in [38]; in [39], a rule extraction method is developed by using ELM and improved ant-miner algorithm for TSA; based on PMU data, reference [40] proposes a TSA method by using kernelized fuzzy rough sets and memetic algorithm for feature selection; in [41], a real-time TSA approach based on EOS-ELM coupled with binary-based feature selection is proposed. In [42], a short-term voltage stability assessment method is proposed by calculating the Lyapunov exponent using PMU data. However, research reports on online monitoring of power system static voltage stability using PMU data have still been relatively rare for a long time. Lim and DeMarco put forward a VSA approach with the use of singular value analysis of PMU data in $[43,44]$. Inspired by these investigations and data-driven decision making $[45,46]$, this paper presents an online static voltage stability analysis method based on PMU data. This method is not limited by the network model and parameters. It only needs to use the active power, reactive power, and voltage amplitude and phase angle measurement collected from PMUs to monitor the static voltage stability of a power system. Finally, the effectiveness of the proposed method is verified by using the IEEE 57-bus system.

\section{Establishment of the Jacobian Matrix Model for Power Flow}

The power flow equation of the power system in polar form can be expressed as follows:

$$
\left\{\begin{array}{l}
P_{i}=V_{i} \sum_{j \in i} V_{j}\left(G_{i j} \cos \theta_{i j}+B_{i j} \sin \theta_{i j}\right), \\
Q_{i}=V_{i} \sum_{j \in i} V_{j}\left(G_{i j} \sin \theta_{i j}-B_{i j} \cos \theta_{i j}\right),
\end{array}\right.
$$

where $j \in i$ denotes that buses $i$ and $j$ are connected; $U_{i}$ and $U_{j}$ represent the voltage amplitude of buses $i$ and $j$, respectively; $G_{i j}$ and $B_{i j}$ represent the values of the real and imaginary parts of the admittance matrix between buses $i$ and $j$, respectively; $\theta_{i j}$ represents the phase angle difference between buses $i$ and $j$; and $P_{i}$ and $Q_{i}$ represent the active and reactive power of bus $i$, respectively.

Excluding the equilibrium bus of the system, according to the Newton-Raphson method, formula (1) can establish the following linearization modified equations:

$$
\left[\begin{array}{c}
\Delta P \\
\Delta Q
\end{array}\right]=\left[\begin{array}{cc}
H_{i j} & N_{i j} \\
J_{i j} & L_{i j}
\end{array}\right]\left[\begin{array}{c}
\Delta \theta \\
\Delta V
\end{array}\right]
$$

where $\Delta P$ and $\Delta Q$ represent the microincrement of active power and reactive power of the injection bus; $\Delta \theta$ and $\Delta V$ represent the microincrement of the voltage phase angle $\theta$ and the voltage amplitude $V$, respectively; $H_{i j}$ and $N_{i j}$ represent the partial derivative of the active power $P$ to the voltage phase angle $\theta$ and the voltage amplitude $V$, respectively; and $J_{i j}$ and $L_{i j}$ represent the partial derivative of the reactive power $Q$ to the voltage phase angle $\theta$ and the voltage amplitude $V$, respectively. In equation (2),

$$
J=\left[\begin{array}{cc}
H_{i j} & N_{i j} \\
J_{i j} & L_{i j}
\end{array}\right],
$$

where $J$ is the Jacobian matrix of the linearized power flow equation.

\section{Evaluation of Voltage Stability by Singular Value Decomposition}

Suppose the system has $n$ buses in addition to the slack bus, of which there are $m$ PV buses, and the singular value decomposition (SVD) $[47,48]$ is performed on equation (3). Then, the following formula can be obtained:

$$
J=E \delta U^{T}=\sum_{i=1}^{(2 n-m)} e_{i} \delta_{i} u_{i}^{T},
$$

where $e_{i}$ and $u_{i}$ represent the $i$-th column elements of $E$ and $U$, respectively, and $\delta_{i}$ is the diagonal element of the diagonal matrix. If the Jacobian matrix is nonsingular, the effect of the increment $\Delta P$ and $\Delta Q$ of the injection powers $P$ and $Q$ on $\theta$ and $V$ can be obtained by equations (2) and (4):

$$
\left[\begin{array}{c}
\Delta \theta \\
\Delta V
\end{array}\right]=J^{-1}\left[\begin{array}{c}
\Delta P \\
\Delta Q
\end{array}\right]=\sum_{i=1}^{2 n-m} \delta_{i}^{-1} u_{i} e_{i}^{T}\left[\begin{array}{c}
\Delta P \\
\Delta Q
\end{array}\right] .
$$

It can be seen from equation (5) that when a singular value $\delta_{i}$ is very small (close to zero), the small change of injection power $P$ and $Q$ will cause great fluctuation of $\theta$ and $V$. The response of the system is completely determined by the minimum singular value (MSV) $\delta_{\min }$ and its corresponding left and right singular vectors $e_{\min }$ and $u_{\min }$, that is:

$$
\left[\begin{array}{c}
\Delta \theta \\
\Delta V
\end{array}\right]=\sum_{i=1}^{2 n-m} \delta_{i}^{-1} u_{\min } e_{\min }^{T}\left[\begin{array}{c}
\Delta P \\
\Delta Q
\end{array}\right]
$$

where $\quad u_{\min }=\left[\theta_{1}, \theta_{2}, \ldots, \theta_{n} ; v_{1}, v_{2}, \ldots, v_{n-m}\right]^{T}$ and $e_{\min }=\left[P_{1}, P_{2}, \ldots, P_{n} ; Q_{1}, Q_{2}, \ldots, Q_{n-m}\right]^{T}$.

Here, $e_{\min }$ and $u_{\min }$ are normalized to 


$$
\begin{aligned}
& \sum_{i=1}^{n} \theta_{i}^{2}+\sum_{i=1}^{n-m} v_{i}^{2}=1 \\
& \sum_{i=1}^{n} P_{i}^{2}+\sum_{i=1}^{n-m} Q_{i}^{2}=1
\end{aligned}
$$

and then

$$
\left[\begin{array}{c}
\Delta P \\
\Delta Q
\end{array}\right]=e_{\min },
$$

and thus

$$
\left[\begin{array}{c}
\Delta \theta \\
\Delta V
\end{array}\right]=\frac{u_{\min }}{\delta_{\min }} .
$$

From equations (8) and (9), it can be concluded that since the MSV is sufficiently small, a small change in the injection power can cause a large change in voltage. The MSV of the Jacobian matrix can be used as a good indicator of static voltage stability $[49,50]$.

\section{Evaluation of Static Voltage Stability by PMU Data}

The aforementioned static voltage stability method by using the MSV of Jacobian matrix can give the static voltage stability quantitatively, but it depends on the fixed physical model and cannot judge the static voltage stability online.

4.1. Estimation of Power Flow Jacobian Matrix by PMU Data. In polar coordinates, the terms of the Jacobian matrix of power flow can be represented by the partial derivative of $\theta_{j}$ and $V_{j}$ by $P_{i}$ and $Q_{i}$, respectively, and then formula (3) can be expressed as

$$
\left\{\begin{array}{l}
H_{i j}=\frac{\partial P_{i}}{\partial \theta_{j}}, \\
N_{i j}=\frac{\partial P_{i}}{\partial V_{j}} V_{j}, \\
J_{i j}=\frac{\partial Q_{i}}{\partial \theta_{j}}, \\
L_{i j}=\frac{\partial Q_{i}}{\partial V_{j}} V_{j} .
\end{array}\right.
$$

Suppose both $\theta_{j}$ and $V_{j}$ have a very small change, represented by $\Delta \theta_{j}$ and $\Delta V_{j}$, respectively. Then, $P_{i}$ and $Q_{i}$ will also change with the slight changes above, denoted by $\Delta P_{i}^{\Delta \theta_{j}}, \Delta P_{i}^{\Delta v_{j}}$ and $\Delta Q_{i}^{\Delta \theta_{j}}, \Delta Q_{i}^{\Delta v_{j}}$, respectively; then, equation (10) can be expressed as [51]

$$
\left\{\begin{array}{l}
H_{i j}=\frac{\partial P_{i}}{\partial \theta_{j}} \approx \frac{\Delta P_{i}^{\Delta \theta_{j}}}{\Delta \theta_{j}}, \\
N_{i j}=\frac{\partial P_{i}}{\partial V_{j}} V_{j} \approx \frac{\Delta P_{i}^{\Delta V_{j}}}{\Delta V_{j}} V_{j}, \\
J_{i j}=\frac{\partial Q_{i}}{\partial \theta_{j}} \approx \frac{\Delta Q_{i}^{\Delta \theta_{j}}}{\Delta \theta_{j}}, \\
L_{i j}=\frac{\partial Q_{i}}{\partial V_{j}} V_{j} \approx \frac{\Delta Q_{i}^{\Delta V_{j}}}{\Delta V_{j}} V_{j} .
\end{array}\right.
$$

At time $t$ :

$$
\left\{\begin{array}{l}
\Delta P_{i}(t) \approx \sum_{j \in N g \cup N_{l}} \Delta P_{i}^{\Delta \theta_{j}}(t)+\sum_{j \in N_{l}} \Delta P_{i}^{\Delta V_{j}}(t), \\
\Delta Q_{i}(t) \approx \sum_{j \in N g \cup N_{l}} \Delta Q_{i}^{\Delta \theta_{j}}(t)+\sum_{j \in N_{l}} \Delta Q_{i}^{\Delta V_{j}}(t),
\end{array}\right.
$$

where $N_{g}$ represents the number of PV buses and $N_{l}$ represents the number of PQ buses, and substituting equation (11) into (12) yields

$$
\left\{\begin{array}{l}
\Delta P_{i}(t) \approx \sum_{j \in N g \cup N_{l}} \Delta \theta_{j}(t) H_{i j}+\sum_{j \in N_{l}} \Delta V_{j}(t) N_{i j}, \\
\Delta Q_{i}(t) \approx \sum_{j \in N g \cup N_{l}} \Delta \theta_{j}(t) J_{i j}+\sum_{j \in N_{l}} \Delta V_{j}(t) L_{i j} .
\end{array}\right.
$$

Further consolidation of equation (13) is available:

$$
\begin{aligned}
& \Delta P_{i} \approx\left[\left(\Delta \theta_{j}\right)_{j \in N g \cup N_{l}}\left(\Delta V_{j}\right)_{j \in N_{l}}\right]\left[\begin{array}{c}
H_{i} \\
N_{i}
\end{array}\right], \\
& \Delta Q_{i} \approx\left[\left(\Delta \theta_{j}\right)_{j \in N g \cup N_{l}}\left(\Delta V_{j}\right)_{j \in N_{l}}\right]\left[\begin{array}{c}
J_{i} \\
L_{i}
\end{array}\right],
\end{aligned}
$$

where $H_{i}=\left[\left(H_{i j}\right), j \in N_{g} \cup N_{l}\right], \quad N_{i}=\left[\left(N_{i j}\right), j \in N_{l}\right]$, $J_{i}=\left[\left(J_{i j}\right), j \in N_{g} \cup N_{l}\right], L_{i}=\left[\left(L_{i j}\right), j \in N_{l}\right]$.

4.2. Model Solution. In the assumption of equations (14) and (15), both the regression matrix and the measurement vector have measurement errors. For the convenience of expression, here we make $A=\left[\left(\Delta \theta_{j}\right)_{j \in N_{g} \cup N_{l}}\left(\Delta \theta_{j}\right)_{j \in N_{l}}\right], b_{i}=P_{i}$; then, equation (14) can be expressed as

$$
b_{i} \approx A\left[\begin{array}{ll}
H_{i}^{T} & N_{i}^{T}
\end{array}\right]^{T} .
$$

Since equation (16) is an overdetermined equation, in this paper, $\left[\begin{array}{ll}H_{i} & N_{i}\end{array}\right]^{T}$ is calculated by using the total least square (TLS) [52, 53]. This method is also suitable for equation (15).

In the ordinary least squares estimate (LSE), since the regression matrix is assumed to be error-free, the principle of this method is to correct $b_{i}$ as little as possible under the 
Euclidean norm [51], which forms the following optimization problem [54]:

$$
\left\{\begin{array}{l}
\min _{\widehat{b_{i}} \in R^{M}}\left\|\Delta b_{i}\right\|_{2}, \\
\widehat{b}_{i}=A\left[\begin{array}{ll}
H_{i}^{T} & N_{i}^{T}
\end{array}\right]^{T},
\end{array}\right.
$$

where $M$ represents sets of synchronized measurements, $\Delta b_{i}=b_{i}-\widehat{b}_{i}\left(\widehat{b}_{i}\right.$ is a very small value), and any $\left[\widehat{H}_{i} \widehat{N}_{i}\right]^{T}$ that satisfies $\widehat{b}_{i}=A\left[\widehat{H}_{i} \widehat{N}_{i}\right]^{T}$ is the solution of LSE of equation (16).

Assuming that $A$ is full rank, the special solution of the closed form of equation (17) is

$$
\left[\widehat{H_{i}} \widehat{N}_{i}\right]^{T}=\left(A^{T} A\right)^{-1} A^{T} b_{i} .
$$

Unlike LSE, TLS also takes into account the measurement error in $A$; similar to the Euclidean norm, the problem is to find a minimized F-norm, as follows:

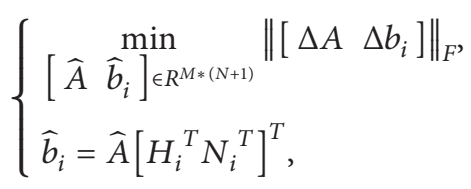

where $\bar{N}=N_{g}+2 N_{l}$ and $\Delta A=A-\widehat{A}, \Delta b_{i}=b_{i}-\widehat{b}_{i}$.

The TLS solution of equation (19) depends on the SVD. We describe this process and write equation (16) as follows:

$$
\left[\begin{array}{ll}
A & b_{i}
\end{array}\right]\left[\begin{array}{lll}
H_{i}^{T} & N_{i}^{T} & -1
\end{array}\right]^{T} \approx 0 .
$$

By using the SVD, the above formula can be rewritten as

$$
\left[\begin{array}{ll}
A & b_{i}
\end{array}\right]=E \delta U^{T} \text {, }
$$

where $E=\left[e_{1}, e_{2}, \ldots, e_{m}\right], U=\left[u_{1}, u_{2}, \ldots, u_{N+1}\right]$ is a symmetric matrix whose diagonal element $\delta_{i}$ is the singular value of $\left[\begin{array}{ll}A & b_{i}\end{array}\right]$. If $\delta_{n+1} \neq 0$; then, the rank of $\left[\begin{array}{ll}A & b_{i}\end{array}\right]$ is $(N+1)$, and the only solution of equation (20) is zero vector. In order to obtain the nonzero solution of equation (20), [ $\left.\begin{array}{ll}A & b_{i}\end{array}\right]$ must be reduced to the rank $N$. The matrix approximation theorem shows that

$$
\left[\begin{array}{ll}
\widehat{A} & \widehat{b_{i}}
\end{array}\right]=\hat{E} \widehat{\delta} U^{T} .
$$

Since the rank of the approximate matrix $\left[\begin{array}{ll}\widehat{A} & \widehat{b_{i}}\end{array}\right]$ is equal to $N$, equation (20) has a nonzero solution. According to the nature of the SVD, $u_{N+1}$ is the unique vector belonging to the zero vector space $\left[\begin{array}{ll}\widehat{A} & \widehat{b}_{i}\end{array}\right]$, and the TLS solution is obtained by scaling the vector $u_{N+1}$ until the last element is -1 :

$$
\left[\begin{array}{lll}
\widehat{H}_{i}^{T} & \widehat{N}_{i}^{T}-1
\end{array}\right]^{T}=-\frac{1}{u_{N+1}^{N+1}} u_{N+1},
$$

where $u_{N+1}^{N+1}$ represents the $(N+1)$ th element of $u_{N+1}$; then, the unique TLS solution of equation (16) is

$$
\left[\begin{array}{ll}
\widehat{H}_{i}^{T} & \widehat{H}_{i}^{T}
\end{array}\right]^{T}=-\frac{1}{u_{\bar{N}+1}^{\bar{N}+1}}\left[u_{\bar{N}+1}^{1}, u_{\bar{N}+1}^{2}, \ldots, u_{\bar{N}+1}^{\bar{N}+1}\right]
$$

\section{Algorithm Flow and Basic Steps}

The flowchart of the proposed method is shown in Figure 1. The specific steps are as follows:

(1) Collect multiple sets of PMU data from different buses at the $(t+\Delta t)$ time, and the number of collected groups is greater than the order of the Jacobian matrix.

(2) Obtain $\Delta P_{i}, \Delta Q_{i}, \Delta \theta_{i}, \Delta V_{i}$, by difference.

(3) Taking the data obtained in step 2 into equation (14), the solution of the overdetermined equations is obtained by the TLS method, and the Jacobian matrix of power flow is obtained.

(4) Calculate the MSV of the Jacobian matrixJobtained in step 3 and compare it with the threshold $\tau$, if the difference exceeds the margin $\xi$.

(5) Repeat steps 1 to 4 to realize real-time monitoring of the power grid.

By collecting the electrical quantities of different buses at time $(t+\Delta t)$, including $P_{\mathrm{i}}, Q_{\mathrm{i}}, \theta_{\mathrm{i}}$, and $V_{\mathrm{i}}$, the power flow Jacobian matrix can be obtained according to equations (14) and (15). This process is shown in Figure 2.

Note that the number of samples is greater than the order of the Jacobian matrix. The results of the MSV of the power flow Jacobian matrix estimated by PMU data and calculated by the Newton-Raphson method are shown in Table 1 .

Comparing the data in Table 1, it can be seen that the difference is small in terms of the MSV obtained by the Newton-Raphson method and the PMU data. This shows that the proposed method can be used to evaluate the static voltage stability of the system.

\section{Case Study}

In this study, it is assumed that all buses of the system are equipped with PMUs. In order to simulate the process of voltage collapse in the power system, the active power $P$ is increased by 0.01 steps in the fifth node of the IEEE 57-bus system, and the MSV of the Jacobian matrix calculated by the proposed method is tracked in real time. The results are shown in Figure 3:

$P / V 8-\delta_{\min }, \quad P / V 13-\delta_{\min }, \quad P / V 31-\delta_{\min }, \quad$ and $P / V 33-\delta_{\min }$ above represent the process of the voltage $V$ changing and the MSV $\delta_{\min }$ of the buses $8,13,31$, and 33 changing with the change of power $P$, respectively. $P / \delta_{\text {min }}$ represents the process in which the MSV of the system varies with power $P$.

It can be seen from the $P / \delta_{\min }$ curve that as the power increases for the different buses, the MSV $\delta_{\text {min }}$ of the system decreases continuously. When $\delta_{\min }$ is close to zero, the system collapses. Comparing the $P / \delta_{\min }$ and $P / V(p u)$ curves, 


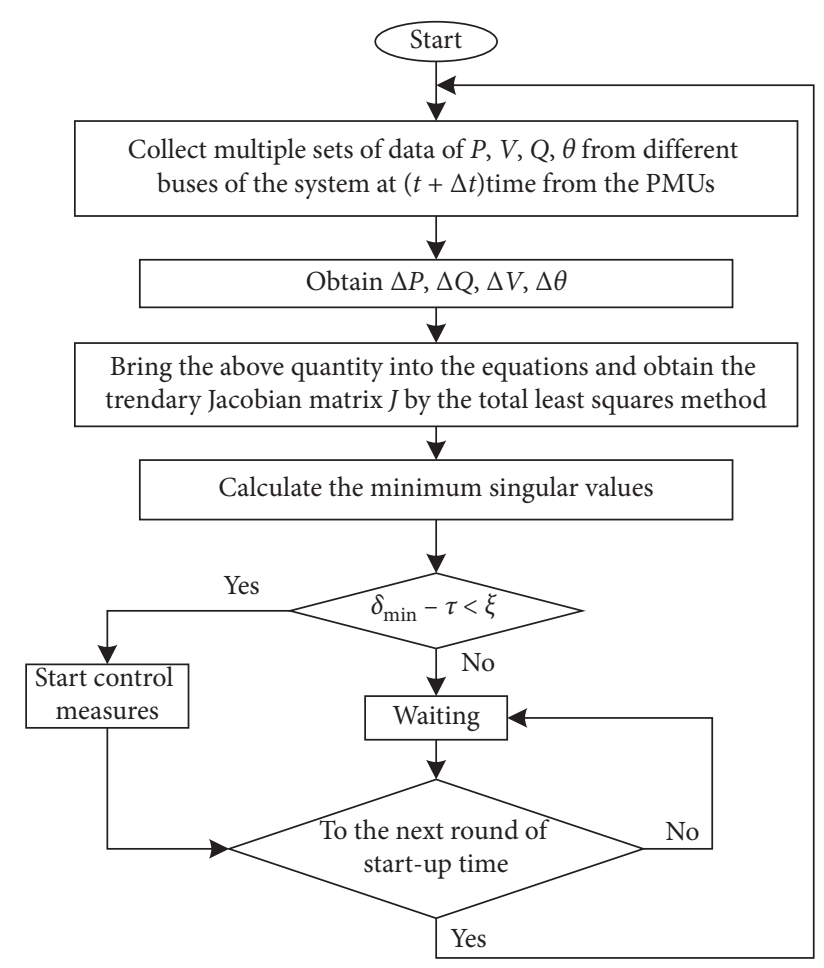

Figure 1: Flowchart of the proposed method.

$$
\begin{aligned}
& \ldots P_{i(k)}, P_{i(k+1)}, \ldots, P_{i(k+x)}, P_{i(k+x+y)} \ldots \\
& \ldots Q_{i(k)}, Q_{i(k+1)}, \ldots, Q_{i(k+x)}, Q_{i(k+x+y)} \ldots \\
& \ldots \theta_{i(k)}, \theta_{i(k+1)}, \ldots, \theta_{i(k+x)}, \theta_{i(k+x+y)} \ldots \\
& \ldots V_{i(k)}, V_{i(k+1)}, \ldots, V_{i(k+x)}, V_{i(k+x+y)} \ldots
\end{aligned}
$$

FIGURE 2: Data collection of PMU.

TABLE 1: MSV based on Newton-Raphson method and PMU data.

\begin{tabular}{lcc}
\hline Bus systems & Newton-Raphson method & PMU data \\
\hline IEEE 9-bus system & 0.8942 & 0.8928 \\
IEEE 14-bus system & 0.5464 & 0.5441 \\
IEEE 30-bus system & 0.2165 & 0.2068 \\
IEEE 57-bus system & 0.1745 & 0.1725 \\
IEEE 118-bus system & 0.1848 & 0.1836 \\
IEEE 300-bus system & 0.0397 & 0.0302 \\
\hline
\end{tabular}

it can be seen that as $\delta_{\min }$ decreases ( $P$ increases continuously), the voltage value also decreases and the rate of decrease is different. When $\delta_{\text {min }}$ is close to zero, a small change of $P$ causes a large fluctuation in voltage, which is consistent with the context in equations (8) and (9).

During normal operation of the two systems, the sampling frequency of PMU is taken as $50 \mathrm{~Hz} / \mathrm{s}$, and the MSV values of the power flow Jacobian matrix are calculated every 4 seconds according to equations (14) and (15). In this way, the evolution curves of the MSV values are demonstrated in Figure 4.

$\delta_{1, \min }$ and $\delta_{2, \min }$ represent the smallest singular value obtained by the Newton-Raphson method and PMU data, respectively. The mean square errors of the two methods are

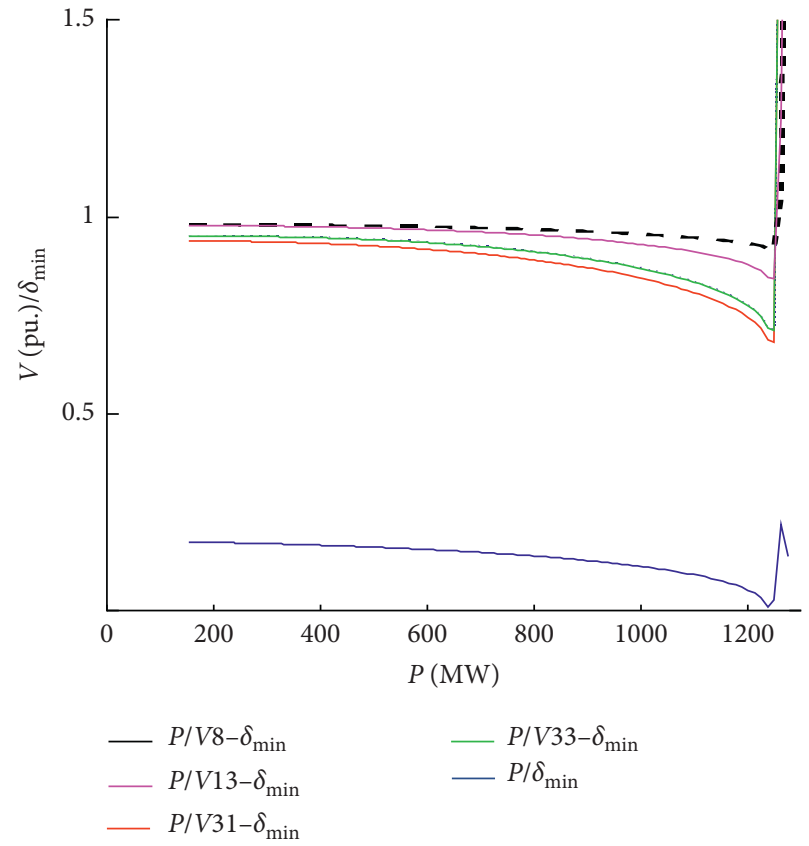

Figure 3: $P-V / \delta$ min curves of the IEEE 57 -bus system.

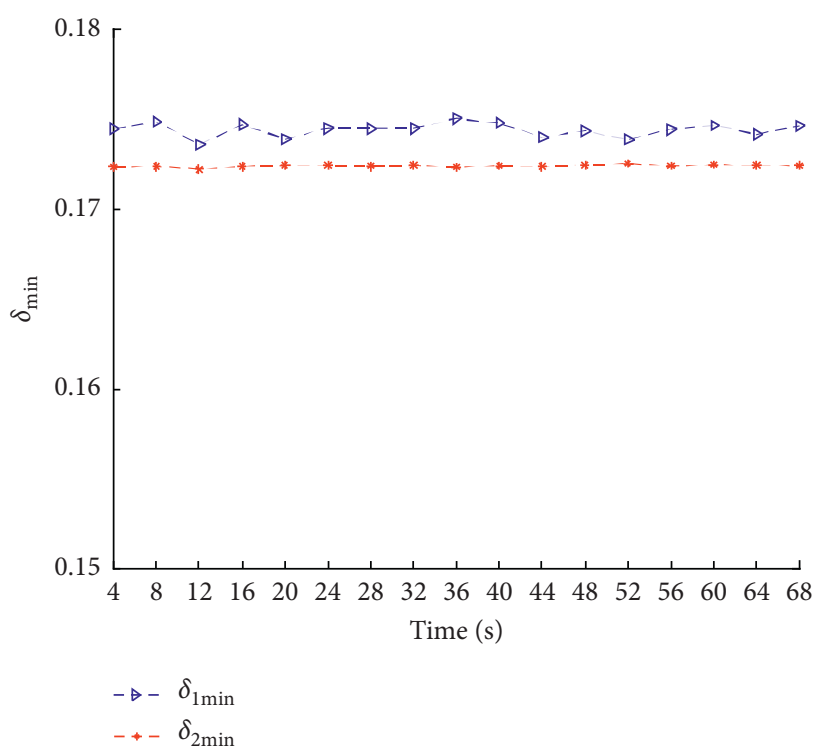

FIgURE 4: Evolution curves of the MSV values in the IEEE 57-bus system.

0.0023 and 0.0002 , respectively. By comparing the matching degree of the curves of Figure 4, it can be seen that the curves obtained by the two methods are basically consistent, indicating that the method proposed in this paper can monitor the static voltage stability of the system online.

The active power $P$ is increased by the step of 0.005 at bus 6 of the IEEE 57-bus system, and the static voltage stability of the power system is estimated from every 200 PMU measurements, as shown in Figure 5.

By comparing the matching degree of the curves in Figure 5, we can see that the curves obtained by the two 


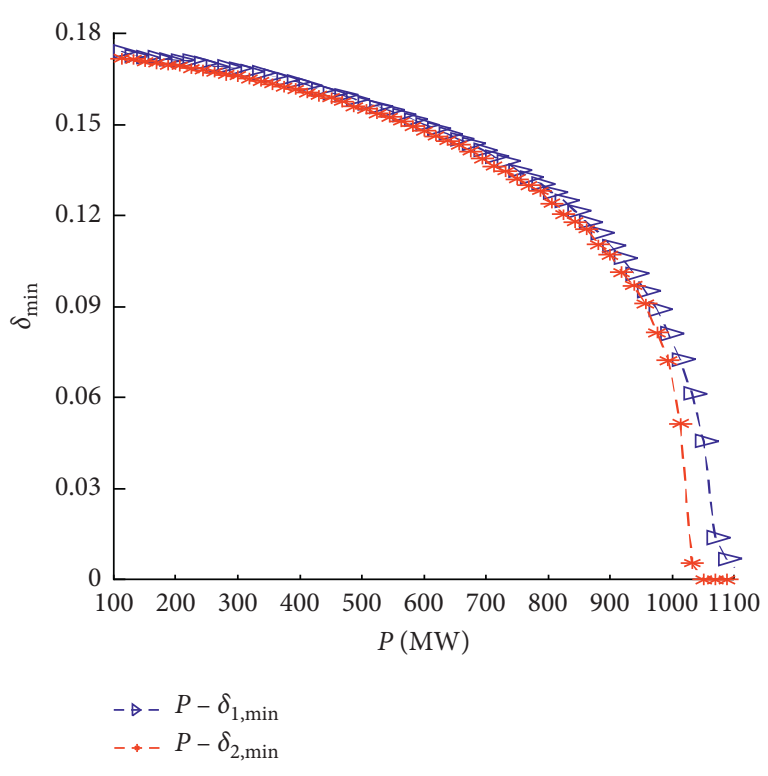

Figure 5: Curves of $P$ - $\delta$ min of IEEE 57 -bus system.

methods are basically consistent, which shows that the method proposed in this paper can realize online monitoring in the process of system instability.

\section{Conclusion}

A method for real-time evaluation of static voltage stability of a power system based on PUM data is proposed. The effectiveness of the proposed method is verified by the IEEE 57-bus system. The conclusions are as follows:

(1) The proposed method is capable of realizing online monitoring of static voltage stability of power systems.

(2) Compared with the traditional method, the proposed method does not need any model and parameter information of the power grid and only needs PMU data for the implementation.

(3) The proposed method can be used in an online stability detection system of power systems for situational awareness improvement.

Our future work will focus on extending the proposed approach to estimate an accurate voltage stability boundary and address stability constraints in voltage stability-constrained optimal power flow [55-58]. It is interesting to investigate the voltage stability of power systems with new elements such as distributed generations [59-61] and combined heat and power plants [62]. Besides, the optimal placement of PMU will be further studied to realize the monitoring of the whole network with fewer PMUs. Another potential topic in future research is to develop voltage stability assessment model using new machine learning techniques such as the lasso algorithm [63], least squares support vector machines [64], and deep learning [65].

\section{Data Availability}

The IEEE 57-bus system data used to support the findings of this study are included within the article.

\section{Conflicts of Interest}

The authors declare that they have no conflicts of interest.

\section{References}

[1] P. M. Anderson and A. A. Fouad, Power System Control and Stability, IEEE, Piscataway, NJ, USA, 2nd edition, 2003.

[2] P. Kundur, J. Paserba, V. Ajjarapu et al., "Definition and classification of power system stability," IEEE Transactions on Industrial Electronics, vol. 19, pp. 1387-1401, 2004.

[3] Y. Li, B. Feng, G. Li, J. Qi, D. Zhao, and Y. Mu, "Optimal distributed generation planning in active distribution networks considering integration of energy storage," Applied Energy, vol. 210, pp. 1073-1081, 2018.

[4] Y. Li, Z. Yang, G. Li, D. Zhao, and W. Tian, "Optimal scheduling of an isolated microgrid with battery storage considering load and renewable generation uncertainties," IEEE Transactions on Industrial Electronics, vol. 66, no. 2, pp. 1565-1575, 2019.

[5] Y. Li, Z. Yang, G. Li et al., "Optimal scheduling of isolated microgrid with an electric vehicle battery swapping station in multi-stakeholder scenarios: a bi-level programming approach via real-time pricing," Applied Energy, vol. 232, pp. 54-68, 2018.

[6] Y. Li, C. Wang, G. Li, J. Wang, D. Zhao, and C. Chen, "Improving operational flexibility of integrated energy system with uncertain renewable generations considering thermal inertia of buildings," Energy Conversion and Management, vol. 207 , p. 112526, 2020.

[7] P. Jin, Y. Li, G. Li, Z. Chen, and X. Zhai, "Optimized hierarchical power oscillations control for distributed generation under unbalanced conditions," Applied Energy, vol. 194, pp. 343-352, 2017.

[8] Y. Li, Y. Li, G. Li, D. Zhao, and C. Chen, "Two-stage multiobjective OPF for AC/DC grids with VSC-HVDC: incorporating decisions analysis into optimization process," Energy, vol. 147, pp. 286-296, 2018.

[9] Y. $\mathrm{Li}$ and S. Wu, "Controlled islanding for a hybrid AC/DC grid with VSC-HVDC using semi-supervised spectral clustering," IEEE Access, vol. 7, pp. 10478-10490, 2019.

[10] B. Feng, X. Zhai, Y. Li, and Z. Wang, "Experimental study on black-start capability of VSC-HVDC for passive networks," in Proceedings of the 2016 PES Asia-Pacific Power \& Energy Engineering Conference (APPEEC), vol. 25-28, pp. 2560-2563, Xi'an, China, October 2016.

[11] Y. Li, Z. Yang, D. Zhao, H. Lei, B. Cui, and S. Li, "Incorporating energy storage and user experience in isolated microgrid dispatch using a multi-objective model," IET Renewable Power Generation, vol. 13, no. 6, pp. 973-981, 2019.

[12] Y. Li and K. Li, "Incorporating demand response of electric vehicles in scheduling of isolated microgrids with renewables using a bi-level programming approach," IEEE Access, vol. 7, pp. 116256-116266, 2019.

[13] Y. Li, Z. Li, and L. Chen, "Dynamic state estimation of generators under cyber attacks," IEEE Access, vol. 7, pp. 125253-125267, 2019.

[14] Z. Qu, Y. Zhang, N. Qu, L. Wang, Y. Li, and Y. Dong, "Method for quantitative estimation of the risk propagation threshold 
in electric power CPS based on seepage probability," IEEE Access, vol. 6, pp. 68813-68823, 2018.

[15] Z. Qu, Q. Xie, Y. Liu et al., "Power cyber-physical system risk area prediction using dependent Markov chain and improved grey wolf optimization," IEEE Access, vol. 8, pp. 82844-82854, 2020.

[16] Y. Li, J. Wang, D. Zhao, G. Li, and C. Chen, "A two-stage approach for combined heat and power economic emission dispatch: combining multi-objective optimization with integrated decision making," Energy, vol. 162, pp. 237-254, 2018.

[17] M. Zhang and Y. Li, "Multi-objective optimal reactive power dispatch of power systems by combining classification-based Multi-objective evolutionary algorithm and integrated decision making," IEEE Access, vol. 8, pp. 38198-38209, 2020.

[18] V. Terzija, G. Valverde, C. Deyu Cai et al., "Wide-area monitoring, protection, and control of future electric power networks," Proceedings of the IEEE, vol. 99, no. 1, pp. 80-93, 2011.

[19] X. Gu and Y. Li, "Transient stability assessment of power systems based on local learning machine and bacterial colony chemotaxis algorithm," Transactions of China Electrotechnical Society, vol. 28, pp. 271-279, 2013.

[20] I. Kamwa, S. R. Samantaray, and G. Joos, "Compliance analysis of PMU algorithms and devices for wide-area stabilizing control of large power systems," IEEE Transactions on Power Systems, vol. 28, no. 2, pp. 1766-1778, 2013.

[21] Y. Li, G. Li, Z. Wang, Z. Han, and X. Bai, "A multifeature fusion approach for power system transient stability assessment using PMU data," Mathematical Problems in Engineering, vol. 2015, p. 1, Article ID 786396, 2015.

[22] M. Glavic and T. Van Cutsem, "Wide-area detection of voltage instability from synchronized phasor measurements. Part II: simulation Results," IEEE Transactions on Power Systems, vol. 24, no. 3, pp. 1417-1425, 2009.

[23] C. D. Vournas, C. Lambrou, and P. Mandoulidis, "Voltage stability monitoring from a transmission bus PMU," IEEE Transactions on Power Systems, vol. 32, no. 4, pp. 3266-3274, 2017.

[24] L. Zhu, C. Lu, and Y. Sun, "Time series shapelet classification based online short-term voltage stability assessment," IEEE Transactions on Power Systems, vol. 31, no. 2, pp. 1430-1439, 2016.

[25] Y. Zhang, Y. Xu, Z. Y. Dong, and R. Zhang, "A hierarchical self-adaptive data-analytics method for real-time power system short-term voltage stability assessment," IEEE Transactions on Industrial Informatics, vol. 15, no. 1, pp. 74-84, 2019.

[26] S. Li, Y. Tan, C. Li, Y. Cao, and L. Jiang, "A fast sensitivitybased preventive control selection method for online voltage stability assessment," IEEE Transactions on Power Systems, vol. 33, no. 4, pp. 4189-4196, 2018.

[27] Z. H. Wang, Z. Y. You, Y. L. Huang et al., "Load margin analysis of hybrid AC/DC system with two terminal VSCHVDC using continuous power flow method," IEEE Transactions on Industrial Informatics, vol. 46, no. 6, pp. 9-15, 2018.

[28] A. O. Ekwue, H. B. Wan, D. T. Y. Cheng, and Y. H. Song, "Singular value decomposition method for voltage stability analysis on the National Grid system (NGC)," International Journal of Electrical Power \& Energy Systems, vol. 21, no. 6, pp. 425-432, 1999.

[29] I. Rahimi Pordanjani and W. Xu, "A singular value decomposition-based technique for decoupling and analyzing power networks," International Journal of Electrical Power \& Energy Systems, vol. 74, pp. 265-273, 2016.
[30] P.-A. Lof, G. Andersson, and D. J. Hill, "Voltage stability indices for stressed power systems," IEEE Transactions on Power Systems, vol. 8, no. 1, pp. 326-335, 1993.

[31] Y. Zhang, Y. Zhang, B. Wu, and J. Zhou, "Power injection model of STATCOM with control and operating limit for power flow and voltage stability analysis," Electric Power Systems Research, vol. 76, no. 12, pp. 1003-1010, 2006.

[32] D. Q. Zhou, U. D. Annakkage, and A. D. Rajapakse, "Online monitoring of voltage stability margin using an artificial neural network," IEEE Transactions on Power Systems, vol. 25, no. 3, pp. 1566-1574, 2010.

[33] H.-Y. Su and C.-W. Liu, "Estimating the voltage stability margin using PMU measurements," IEEE Transactions on Power Systems, vol. 31, no. 4, pp. 3221-3229, 2016.

[34] P. Zhang, F. Li, and N. Bhatt, "Next-generation monitoring, analysis, and control for the future smart control center," IEEE Transactions on Smart Grid, vol. 1, no. 2, pp. 186-192, 2010.

[35] Y. Zhang, T. Li, G. Na, G. Li, and Y. Li, "Optimized extreme learning machine for power system transient stability prediction using synchrophasors," Mathematical Problems in Engineering, vol. 2015, p. 1, Article ID 529724, 2015.

[36] Y. Li and X. Gu, "Power system transient stability assessment based on online sequential extreme learning machine," in Proceedings of the 2013 PES Asia-Pacific Power \& Energy Engineering Conference (APPEEC), vol. 2560-2563, pp. 1-4, Hong Kong, China, December 2013.

[37] Y. Li and X. Gu, "Feature selection for transient stability assessment based on improved maximal relevance and minimal redundancy criterion," Proceedings of the CSEE, vol. 33, pp. 179-186, 2013.

[38] Y. Li, G. Li, and X. Gu, "Transient stability assessment of power systems based on ensemble OS-ELM," Transactions of China Electrotechnical Society, vol. 30, pp. 412-418, 2015.

[39] Y. Li, G. Li, and Z. Wang, "Rule extraction based on extreme learning machine and an improved ant-miner algorithm for transient stability assessment," PLoS ONE, vol. 10, no. 6, 2015.

[40] X. Gu, Y. Li, and J. Jia, "Feature selection for transient stability assessment based on kernelized fuzzy rough sets and memetic algorithm," International Journal of Electrical Power \& Energy Systems, vol. 64, pp. 664-670, 2015.

[41] Y. Li and Z. Yang, "Application of EOS-ELM with binary Jayabased feature selection to real-time transient stability assessment using PMU data," IEEE Access, vol. 5, pp. 2309223101, 2017.

[42] S. Dasgupta, M. Paramasivam, U. Vaidya, and V. Ajjarapu, "Real-Time monitoring of short-term voltage stability using PMU data," IEEE Transactions on Power Systems, vol. 28, no. 4, pp. 3702-3711, 2013.

[43] J. M. Lim and C. L. DeMarco, "Model-free voltage stability assessments via singular value analysis of PMU data," Power Grid, vol. 28, pp. 1-10, 2013.

[44] J. M. Lim and C. L. Demarco, "SVD-based voltage stability assessment from phasor measurement unit data," IEEE Transactions on Power Systems, vol. 31, no. 4, pp. 2557-2565, 2016.

[45] Z.-B. Shi, T. Yu, Q. Zhao, Y. Li, and Y.-B. Lan, "Comparison of algorithms for an electronic nose in identifying liquors," Journal of Bionic Engineering, vol. 5, no. 3, pp. 253-257, 2008.

[46] H. Lan, T. Ai, and Y. Li, "Application of EMD on single-pole adaptive reclosure of transmission," Power System Protection and Control, vol. 38, no. 12, pp. 35-39, 2010.

[47] K. Ben-Kilani and M. Elleuch, "Structural analysis of voltage stability in power systems integrating wind power," IEEE 
Transactions on Power Systems, vol. 28, no. 4, pp. 3785-3794, 2013.

[48] M. Dehghani, B. Shayanfard, and A. R. Khayatian, "PMU ranking based on singular value decomposition of dynamic stability matrix," IEEE Transactions on Power Systems, vol. 28, no. 3, pp. 2263-2270, 2013.

[49] A. Tiranuchit and R. J. Thomas, "A posturing strategy against voltage instabilities in electric power systems," IEEE Transactions on Power Systems, vol. 3, no. 1, pp. 87-93, 1988.

[50] O. A. Urquidez and L. Xie, "Singular value sensitivity based optimal control of embedded VSC-HVDC for steady-state voltage stability enhancement," IEEE Transactions on Power Systems, vol. 31, no. 1, pp. 216-225, 2016.

[51] Y. C. Chen, J. Wang, A. D. Domínguez-García, and P. W. Sauer, "Measurement-based estimation of the power flow Jacobian matrix," IEEE Transactions on Smart Grid, vol. 7, no. 5, pp. 2507-2515, 2016.

[52] H.-A. Diao, Y. Wei, and P. Xie, "Small sample statistical condition estimation for the total least squares problem," Numerical Algorithms, vol. 75, no. 2, pp. 435-455, 2016.

[53] A. M. Ibrahim, M. I. Marei, S. F. Mekhamer, and M. M. Mansour, "An artificial neural network based protection approach using total least square estimation of signal parameters via the rotational invariance technique for flexible AC transmission system compensated transmission lines," Electric Power Components and Systems, vol. 39, no. 1, pp. 64-79, 2011.

[54] L. Xu and F. Ding, "Recursive least squares and multi-innovation stochastic gradient parameter estimation methods for signal modeling," Circuits, Systems, and Signal Processing, vol. 36, no. 4, pp. 1735-1753, 2017.

[55] Y. Li and Y. Li, "Two-step many-objective optimal power flow based on knee point-driven evolutionary algorithm," Processes, vol. 6, no. 12, p. 250, 2018.

[56] Y. Li, Y. Li, and G. Li, "A multi-objective optimal power flow approach considering economy and environmental factors for hybrid AC/DC grids incorporating VSC-HVDC," Power System Technology, vol. 40, pp. 2661-2667, 2016.

[57] Y. Li, Y. Li, and G. Li, "A two-stage multi-objective optimal power flow algorithm for hybrid AC/DC grids with VSCHVDC," in Proceedings of the 2017 IEEE Power \& Energy Society General Meeting, pp. 1-5, Chicago, IL, USA, July 2017.

[58] Y. Li and Y. Li, "Security-constrained multi-objective optimal power flow for a hybrid AC/VSC-MTDC system with lassobased contingency filtering," IEEE Access, vol. 8, pp. 68016811, 2020.

[59] K. Kawabe, Y. Ota, A. Yokoyama, and K. Tanaka, "Novel dynamic voltage support capability of photovoltaic systems for improvement of short-term voltage stability in power systems," IEEE Transactions on Power Systems, vol. 32, no. 3, pp. 1796-1804, 2017.

[60] U. Sultana, A. B. Khairuddin, M. M. Aman, A. S. Mokhtar, and N. Zareen, "A review of optimum DG placement based on minimization of power losses and voltage stability enhancement of distribution system," Renewable and Sustainable Energy Reviews, vol. 63, pp. 363-378, 2016.

[61] E. Vittal, M. O'Malley, and A. Keane, "A steady-state voltage stability analysis of power systems with high penetrations of wind," IEEE Transactions on Power Systems, vol. 25, no. 1, pp. 433-442, 2010.

[62] J. Sun and Y. Li, "Social cognitive optimization with tent map for combined heat and power economic dispatch," International Transactions on Electrical Energy Systems, vol. 29, no. 1, p. e2660, 2018.
[63] Y. Li, Y. Li, and Y. Sun, "Online static security assessment of power systems based on lasso algorithm," Applied Sciences, vol. 8, no. 9, p. 1442, 2018.

[64] Z. B. Shi, Y. Li, and T. Yu, "Short-term load forecasting based on LS-SVM optimized by bacterial colony chemotaxis algorithm," in Proceedings of the 2009 International Conference on Information and Multimedia Technology, pp. 306-309, Jeju Island, South Korea, December 2009.

[65] Y. LeCun, Y. Bengio, and G. Hinton, "Deep learning," Nature, vol. 521, no. 7553, pp. 436-444, 2015. 